THE Astrophysical Journal, 478:585-593, 1997 April 1

(C) 1997. The American Astronomical Society. All rights reserved. Printed in U.S.A.

\title{
THE FORMATION OF LARGE HYDROCARBONS AND CARBON CLUSTERS IN DENSE INTERSTELLAR CLOUDS
}

\author{
R. P. A. BETTENS ${ }^{1}$ \\ Department of Physics, The Ohio State University, Columbus, OH 43210-1106 \\ AND \\ ERIC HERBST \\ Departments of Physics and Astronomy, The Ohio State University, Columbus, OH 43210-1106 \\ Received 1996 September 5 ; accepted 1996 October 23
}

\begin{abstract}
Current gas-phase models of dense cloud chemistry contain molecules through $\approx 10$ atoms in size. We have extended two current reaction networks to include unsaturated hydrocarbons and bare carbon clusters up to 64 carbon atoms in size. The new molecules consist of linear, monocyclic, tricyclic, and fullerene/ane species, but not polycyclic aromatic hydrocarbons (PAHs). In an earlier paper, which was directed at a possible understanding of the diffuse interstellar absorption band problem, we reported results for dispersive clouds, which start as dense clouds but evolve into diffuse material. In this paper, we report calculated molecular abundances for homogeneous dense interstellar clouds. The chemistry of linear unsaturated species, especially the $\mathrm{C}_{n} \mathrm{H}$ radicals, is emphasized. As with their smaller organic counterparts, the newly included large molecules possess abundances that mainly peak at so-called early times before declining to steady state values. Exceptions include the stable fullerene $\mathrm{C}_{60}$, which rises to a maximum abundance at steady state. The results for large molecules in dense clouds are highly dependent on which model network is used.
\end{abstract}

Subject headings: ISM: abundances - ISM: clouds - ISM: molecules - molecular processes

\section{INTRODUCTION}

The largest gaseous molecule detected unambiguously in dense interstellar clouds $\left(\mathrm{HC}_{9} \mathrm{~N}\right)$ contains 11 atoms (Herbst 1995; Travers et al. 1996). Models of dense cloud chemistry include a wide assortment of molecules up to approximately this size (Herbst \& Leung 1989, 1990; Bettens, Lee, \& Herbst 1995; Lee, Bettens, \& Herbst 1996a), with abundances in non-star-forming regions most often calculated as a function of time with nonvarying physical conditions. These so-called "pseudo-time-dependent" models are normally run for homogeneous conditions, although in recent years inhomogeneities have begun to be incorporated by the use of slabs (Lee et al. 1996b; Xie, Allen, \& Langer 1995; Bergin, Langer, \& Goldsmith 1995). For the larger molecules, the calculated abundances in homogeneous models are very sensitive to both time and to what neutral-neutral reactions are included with large rates at low temperature (Bettens et al. 1995). When the initial species are assumed to be neutral or singly charged atoms (with the exception of $\mathrm{H}_{2}$ ), the abundances of organic molecules typically rise until they peak at a so-called "early time" of $10^{3}-10^{5} \mathrm{yr}$, before declining sharply to steady state values, which are reached in $10^{7}-10^{8} \mathrm{yr}$. It is the early-time values that are more often used in comparison with observations. The inclusion of large numbers of rapid neutral-neutral reactions (Herbst et al. 1994; Bettens et al. 1995), based on a few laboratory experiments (Sims \& Smith 1995), leads to strong reductions in the peak early-time abundances, generally worsening agreement between calculated values and observed abundances. Lee et al. (1996a) have tabulated large numbers of results for three model networks - the "new standard model," the "new neutral-neutral model,"

\footnotetext{
${ }^{1}$ Current address: Research School of Chemistry, Australian National University, Canberra, ACT 0200, Australia.
}

and "Model 4 " (Bettens et al. 1995). The first network contains a rather limited set of rapid neutral-neutral reactions, the second contains a much larger set, while the third contains most of the reactions included in the new neutralneutral model but omits rapid reactions between $\mathrm{O}$ and $\mathrm{N}$ atoms with bare carbon clusters. In general, the early-time results using Model 4 (hereafter M4) are rather similar to those of the new standard model (NSM) in that they show large abundances of organic molecules, although the NSM results are still in better agreement with observations of dense clouds. Some justification for omitting obviously crucial reactions between $\mathrm{O}$ and carbon clusters has been provided by ab initio calculations on $\mathrm{O}+\mathrm{C}_{3}$ (Woon \& Herbst 1996).

In a recent series of papers (Bettens \& Herbst 1995, 1996), we reported the extension of our two successful model networks - NSM and M4 - to include molecules far larger than heretofore contained, and we presented calculations of the abundances of these large molecules in the diffuse interstellar medium. The M4 model was also refined by reintroducing the rapid reaction between $\mathrm{O}$ and $\mathrm{C}_{2}$, which had been removed from the new neutral-neutral network along with the $\mathrm{O}+\mathrm{C}_{n>2}$ reactions. The additional molecules range up to 64 carbon atoms in size and include bare carbon clusters, as well as very unsaturated (hydrogenpoor) hydrocarbons that contain up to two hydrogen atoms for neutral species and three hydrogen atoms for ionic species. In addition to neutral species and positive ions, selected negative ions are included. Despite the existence of two small ring molecules in interstellar clouds, the new species are assumed for simplicity to be linear when the number of carbon atoms is less than or equal to 23 , at which stage it is assumed that spontaneous conversion occurs to form monocylic ring species. The particular size is chosen because each C-C-C group needs to bend only about $15^{\circ}$ for cyclization to occur. The laboratory identification of the 
linear $\mathrm{C}_{13}$ isomer shows that carbon clusters of this size are not yet large enough to spontaneously convert to rings (Giesen et al. 1994). The possibility that chemical reactions can interconvert linear and cyclic isomers has not been considered. Once formed, monocyclic rings then lead to tricyclic rings and finally fullerenes, especially $\mathrm{C}_{60}$. Partially hydrogenated neutral fullerenes ("fulleranes") have not been included in the model but may well exist, so that our calculated abundances of neutral fullerenes should be considered to be the sum of fullerenes and fulleranes.

Details of the reaction networks, which contain about 1000 species and 10,000 reactions, are in our first paper (Bettens \& Herbst 1995). In the networks, the growth of linear and monocyclic species occurs mainly through $\mathrm{C}$ and $\mathrm{C}^{+}$insertion and association reactions; in the latter, the two reactants combine to form one larger product. In general, association reactions become more important and normal reactions less important as the size of the reactants increases. Tricyclic species are formed through association and condensation of monocyclic species, and fullerenes are formed via conversion reactions between small ions and tricyclic rings. Both sets of latter species grow by association with $\mathrm{C}_{\text {and }} \mathrm{C}^{+}$. The synthesis of the large molecules is based on analogous laboratory work (von Helden, Gotts, \& Bowers 1993; Hunter et al. 1994) leading to fullerene production. Many of the needed rate coefficients and product branching fractions were estimated using a variation on the Rice-Ramsberger-Kassel-Marcus (RRKM) statistical technique (Bettens \& Herbst 1995; Herbst 1996).

In our earlier work on the diffuse interstellar medium (Bettens \& Herbst 1996), we found that large molecules could be produced efficiently in dispersive clouds. The work was motivated in part by a more simplified model of large molecule growth in the diffuse interstellar medium proposed by Thaddeus (1994). Dispersive clouds start off life as dense clouds, in which gas-phase chemistry can lead to sizeable abundances of hydrocarbons that are moderate in size, known as "seeds." Once the cloud expands and the density is reduced beyond a certain value, external photons lead to the production of large abundances of atomic carbon, which enhances the production of large molecules from the seeds. Eventually, significant abundances of very large molecules-mainly monocyclic rings and fullerenes stable against photodissociation by the interstellar radiation field - are formed. The specific results depend significantly on which model is being extended; the M4 results generally show much higher abundances of complex species, although photons enhance large molecule production significantly and rapidly in the NSM model. Large abundances of linear hydrocarbons and clusters under diffuse conditions are not possible in either model unless an additional steady supply of seed molecules can be photodesorbed off interstellar grains to lead to their production. The various results indicate that an assortment of large molecules can plausibly be regarded as candidates for carriers of the diffuse interstellar bands (DIBs) (Herbig 1993).

In this paper, we concentrate on the chemistry of large hydrocarbons and carbon clusters in dense interstellar clouds, a subject we have tackled before in a much less detailed fashion (Herbst 1991). The extensions to our NSM and M4 model networks have been used to calculate the pseudo-time-dependent chemistry in a cloud of gas density $n_{\mathrm{H}}=n(\mathrm{H})+2 n\left(\mathrm{H}_{2}\right)=2 \times 10^{4} \mathrm{~cm}^{-3}$ and temperature $10 \mathrm{~K}$. The elemental abundances, the cosmic ray ionization



FIG. 1.-Plot of the fractional abundances obtained with the NSM network for the linear species $1-\mathrm{C}_{n}, 1-\mathrm{C}_{n} \mathrm{H}$, and $1-\mathrm{C}_{n} \mathrm{H}_{2}$ vs. the number of carbon atoms $n$ at a time of $10^{4.5} \mathrm{yr}$.

rate, and the initial nonzero concentrations are our standard values and have been discussed previously (Lee et al. 1996a). Since the elemental abundances used are the "lowmetal" values, only one solution-the low-ionization phase - is known to exist (Le Bourlot, Pineau des Forêts, \& Roueff 1995).

\section{RESULTS: LINEAR SPECIES}

Figures 1 and 2 show for the extended NSM and M4 networks, respectively, the fractional abundances (with respect to $\mathrm{H}_{2}$ ) of the linear neutral species $\mathrm{C}_{n}, \mathrm{C}_{n} \mathrm{H}$, and $\mathrm{C}_{n} \mathrm{H}_{2}(n \leq 23)$ as a function of the number of carbon atoms $n$ at a specific early time $\left(10^{4.5} \mathrm{yr}\right)$. No distinction is made in our model results between acetylene-type structures $\left(\mathrm{HC}_{n} \mathrm{H}\right)$, which are nonpolar, and carbene structures $\left(\mathrm{H}_{2} \mathrm{C}_{n}\right)$, which are polar and therefore detectable by radio astrono-

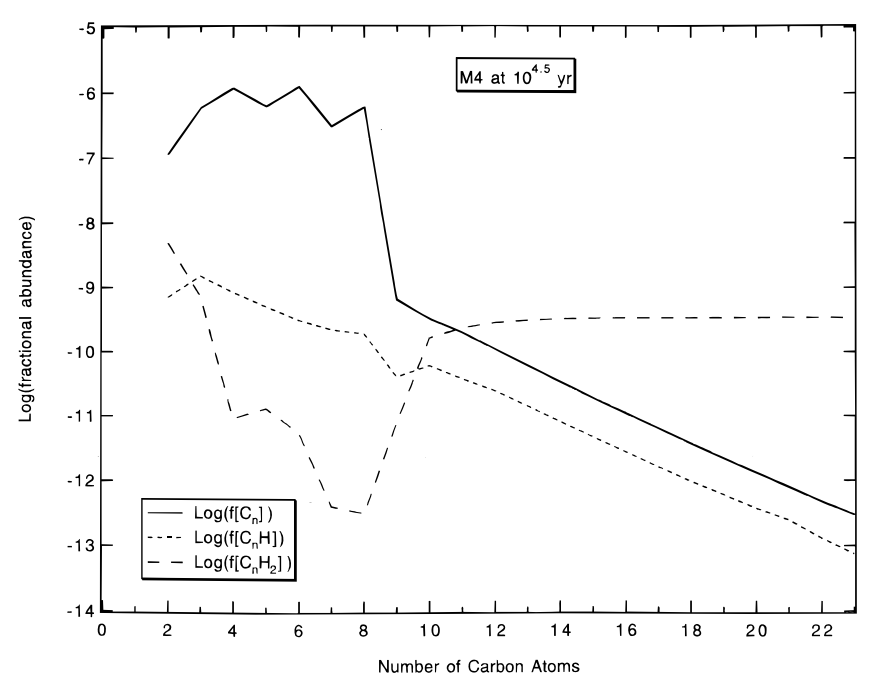

Fig. 2.-Plot of the fractional abundances obtained with the M4 network for the linear species $1-\mathrm{C}_{n}, 1-\mathrm{C}_{n} \mathrm{H}$, and $1-\mathrm{C}_{n} \mathrm{H}_{2}$ vs. the number of carbon atoms $n$ at a time of $10^{4.5} \mathrm{yr}$. 
mical methods. In our kinetic calculations, however, the more stable acetylene-type isomers were used. One can see immediately that the diminution in abundance with increasing $n$ is far larger for the NSM network than for the M4 network, which is also more powerful synthetically in dispersive clouds (Bettens \& Herbst 1996). For the former network, the fractional abundance of $\mathrm{C}_{20} \mathrm{H}$, for example, is less than $10^{-16}$, whereas for the latter work it exceeds $10^{-13}$. In addition, there is little if any diminution in the predicted abundances of the $\mathrm{C}_{n} \mathrm{H}_{2}$ species with increasing $n$ in the M4 predictions; a rather constant fractional abundance of $\approx 3 \times 10^{-10}$ for $n \geq 10$ is predicted. An actual increase in abundance occurs with increasing $n$ in the range 8-10 for both models because of a greater ease in hydrogenation. Table 1 gives the fractional abundances for the $\mathrm{C}_{n} \mathrm{H}$ radicals at the same early time as depicted in Figures 1 and 2 ; in addition, fractional abundances for the negative ions of these species are also listed. These negative ions, which are formed for $n \geq 10$ by direct attachment of electrons to neutral radicals and by direct attachment of electrons to bare clusters followed by reactions with atomic hydrogen (Bettens \& Herbst 1996), are among the most abundant negatively charged species in our models because of the large estimated electron affinities of the neutral molecules.

In Figures 3-8, we show three-dimensional plots of the fractional abundances of the three types of linear neutral species $\left(\mathrm{C}_{n}, \mathrm{C}_{n} \mathrm{H}\right.$, and $\left.\mathrm{C}_{n} \mathrm{H}_{2}\right)$ versus both time and number of carbon atoms $n$. Figures 3,4 , and 5 refer to the NSM network, while Figures 6, 7, and 8 refer to the M4 network. In these and subsequent three-dimensional figures, the abundance is shown on a gray scale, with darker shades of gray referring to lower abundances. Note that Figures 1 and
TABLE 1

Fractional Abundances with Respect to $\mathrm{H}_{2}$ at $10^{4.5}$ Years for Linear $\mathrm{C}_{n} \mathrm{H}$ Neutral Radicals and Negative Ions, Using NSM AND M4 NETWORKS

\begin{tabular}{ccccc}
\hline \hline$n$ & $\mathrm{C}_{n} \mathrm{H}(\mathrm{NSM})$ & $\mathrm{C}_{n} \mathrm{H}(\mathrm{M} 4)$ & $\mathrm{C}_{n} \mathrm{H}^{-}(\mathrm{NSM})$ & $\mathrm{C}_{n} \mathrm{H}^{-}(\mathrm{M} 4)$ \\
\hline $2 \ldots \ldots$ & $1.3(-7)$ & $7.1(-10)$ & $\ldots$ & $\ldots$ \\
$3 \ldots \ldots$ & $5.6(-8)$ & $1.5(-9)$ & $\ldots$ & $\ldots$ \\
$4 \ldots \ldots$ & $1.5(-7)$ & $8.3(-10)$ & $\ldots$ & $\ldots$ \\
$5 \ldots \ldots$ & $7.9(-9)$ & $5.0(-10)$ & $\ldots$ & $\ldots$ \\
$6 \ldots \ldots$ & $4.2(-9)$ & $3.1(-10)$ & $\ldots$ & $\ldots$ \\
$7 \ldots \ldots$ & $1.9(-9)$ & $2.2(-10)$ & $\ldots$ & $\ldots$ \\
$8 \ldots \ldots$ & $2.5(-9)$ & $1.9(-10)$ & $\ldots$ & $\ldots$ \\
$9 \ldots \ldots$ & $2.5(-10)$ & $4.1(-11)$ & $\ldots$ & $\ldots$ \\
$10 \ldots \ldots$ & $1.3(-11)$ & $6.2(-11)$ & $3.2(-13)$ & $2.3(-12)$ \\
$11 \ldots \ldots$ & $7.1(-12)$ & $3.8(-11)$ & $1.8(-13)$ & $1.4(-12)$ \\
$12 \ldots \ldots$ & $2.1(-12)$ & $2.5(-11)$ & $5.1(-14)$ & $9.2(-13)$ \\
$13 \ldots \ldots$ & $6.5(-13)$ & $1.5(-11)$ & $1.6(-14)$ & $5.4(-13)$ \\
$14 \ldots \ldots$ & $1.8(-13)$ & $8.4(-12)$ & $4.4(-15)$ & $3.1(-13)$ \\
$15 \ldots \ldots$ & $5.1(-14)$ & $4.9(-12)$ & $1.3(-15)$ & $1.8(-13)$ \\
$16 \ldots \ldots$ & $1.4(-14)$ & $2.8(-12)$ & $3.3(-16)$ & $1.0(-13)$ \\
$17 \ldots \ldots$ & $3.9(-15)$ & $1.7(-12)$ & $9.6(-17)$ & $6.1(-14)$ \\
$18 \ldots \ldots$ & $1.0(-15)$ & $9.7(-13)$ & $2.5(-17)$ & $3.6(-14)$ \\
$19 \ldots \ldots$ & $3.0(-16)$ & $6.0(-13)$ & $7.3(-18)$ & $2.2(-14)$ \\
$20 \ldots \ldots$ & $7.8(-17)$ & $3.7(-13)$ & $1.9(-18)$ & $1.4(-14)$ \\
$21 \ldots \ldots$ & $2.3(-17)$ & $2.4(-13)$ & $5.7(-19)$ & $9.0(-15)$ \\
$22 \ldots \ldots$ & $6.2(-18)$ & $1.3(-13)$ & $1.5(-19)$ & $4.8(-15)$ \\
$23 \ldots \ldots$ & $1.9(-18)$ & $7.5(-14)$ & $4.6(-20)$ & $2.8(-15)$ \\
\hline
\end{tabular}

2 represent cuts of the three-dimensional figures at a fixed early time. The three-dimensional figures indicate, however, that the general features of Figures 1 and 2 are reproduced at a wide variety of early times. The most striking feature about the three-dimensional curves, which are rather complex, is that they show the linear neutral species to reach maximum abundances very quickly compared with


FIG. 3.-Three-dimensional plot of the fractional abundance of the linear species $1-C_{n}$ vs. $n$ and time obtained with the NSM network. The gray scale is used in conjunction with the ordinate to indicate fractional abundance. 


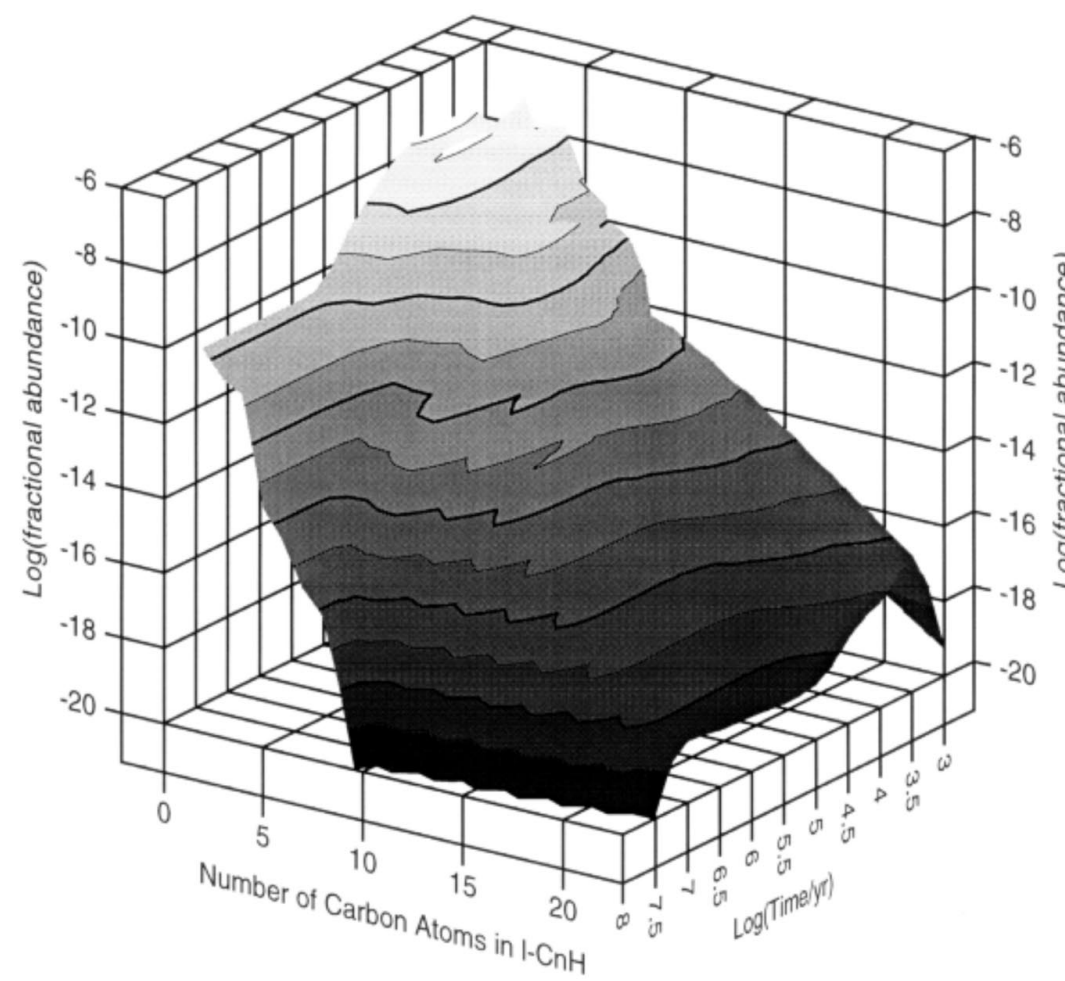

$-6$

$-10$

$-12$

$-14$

$-16$

$-18$

FIG. 4. - Three-dimensional plot of the fractional abundance of the linear species $1-\mathrm{C}_{n} \mathrm{H}$ vs. $n$ and time obtained with the NSM network. The gray scale is used in conjunction with the ordinate to indicate fractional abundance.

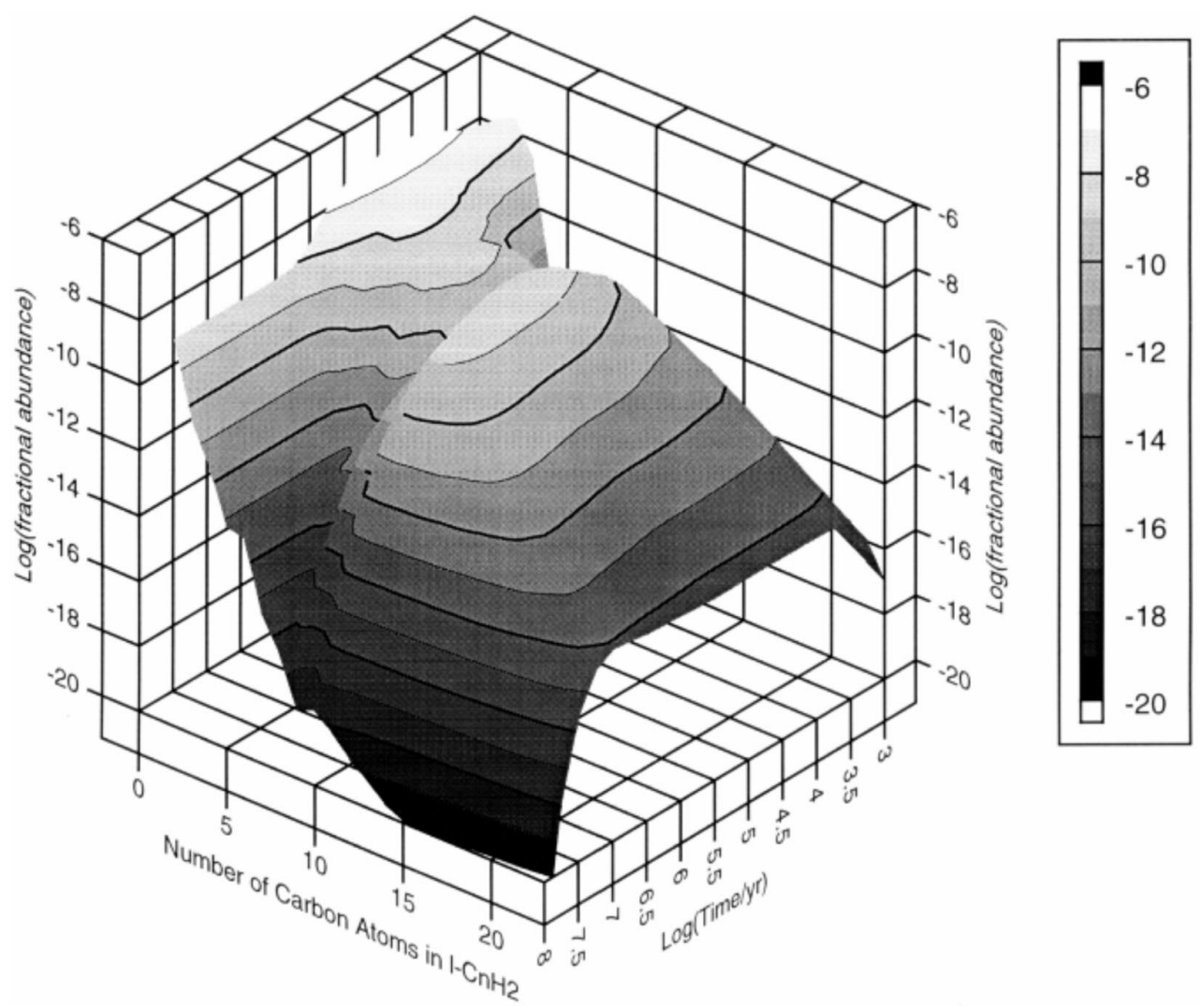

FIG. 5.-Three-dimensional plot of the fractional abundance of the linear species $1-\mathrm{C}_{n} \mathrm{H}_{2}$ vs. $n$ and time obtained with the NSM network. The gray scale is used in conjunction with the ordinate to indicate fractional abundance. 


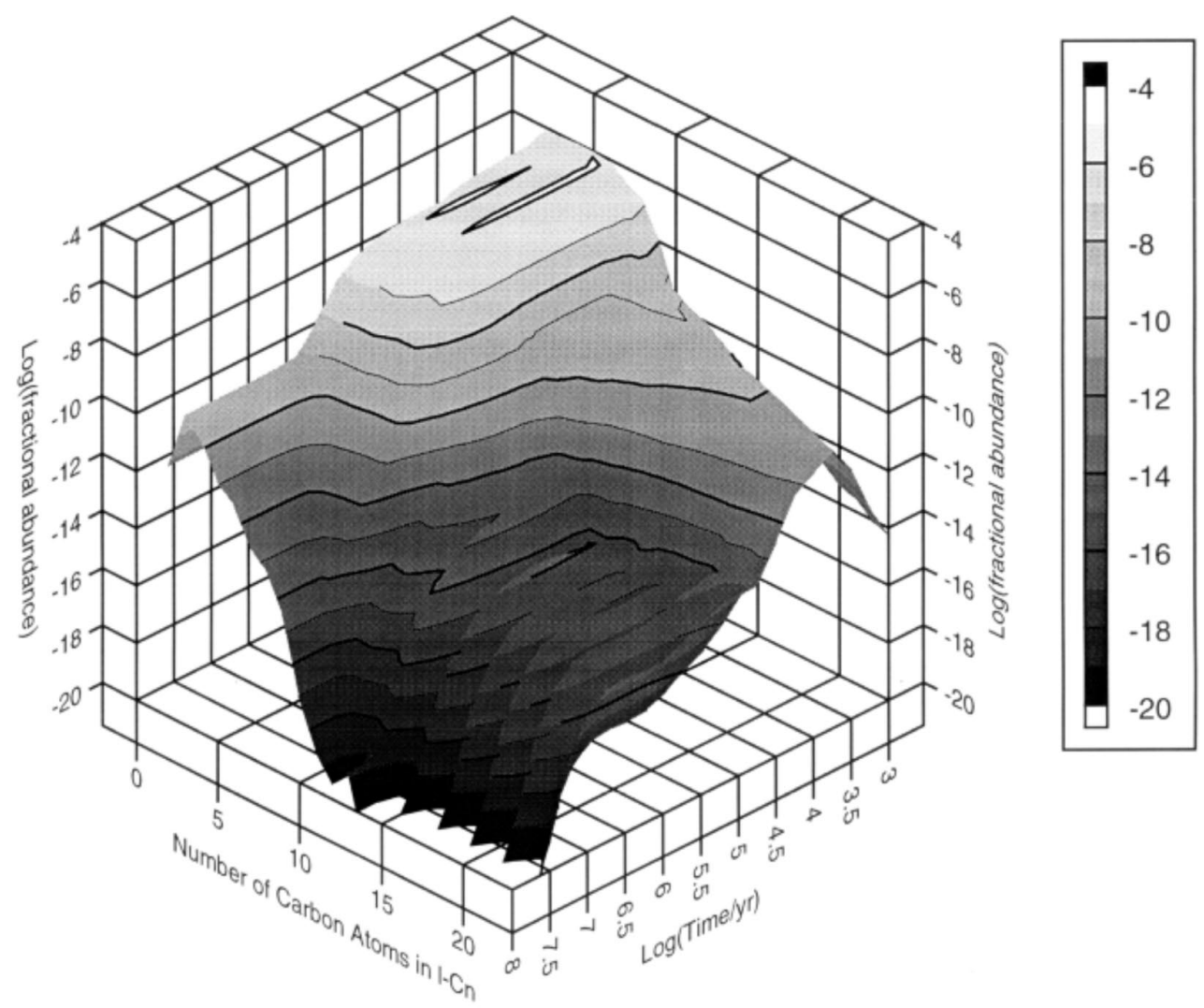

FIG. 6.-Three-dimensional plot of the fractional abundance of the linear species $1-\mathrm{C}_{n}$ vs. $n$ and time obtained with the M4 network. The gray scale is used in conjunction with the ordinate to indicate fractional abundance.

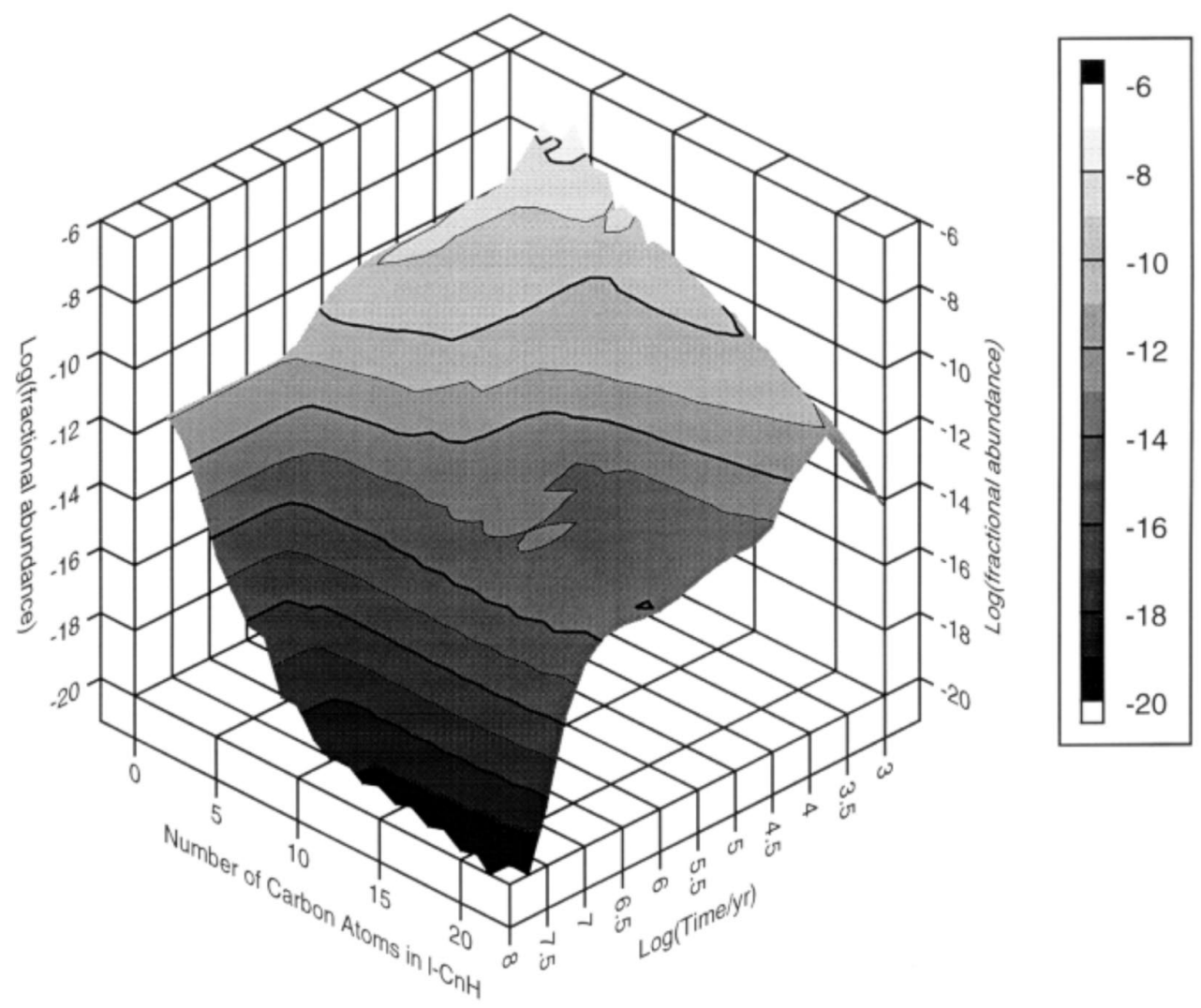

Fig. 7.- Three-dimensional plot of the fractional abundance of the linear species $1-\mathrm{C}_{n} \mathrm{H}$ vs. $n$ and time obtained with the M4 network. The gray scale is used in conjunction with the ordinate to indicate fractional abundance. 


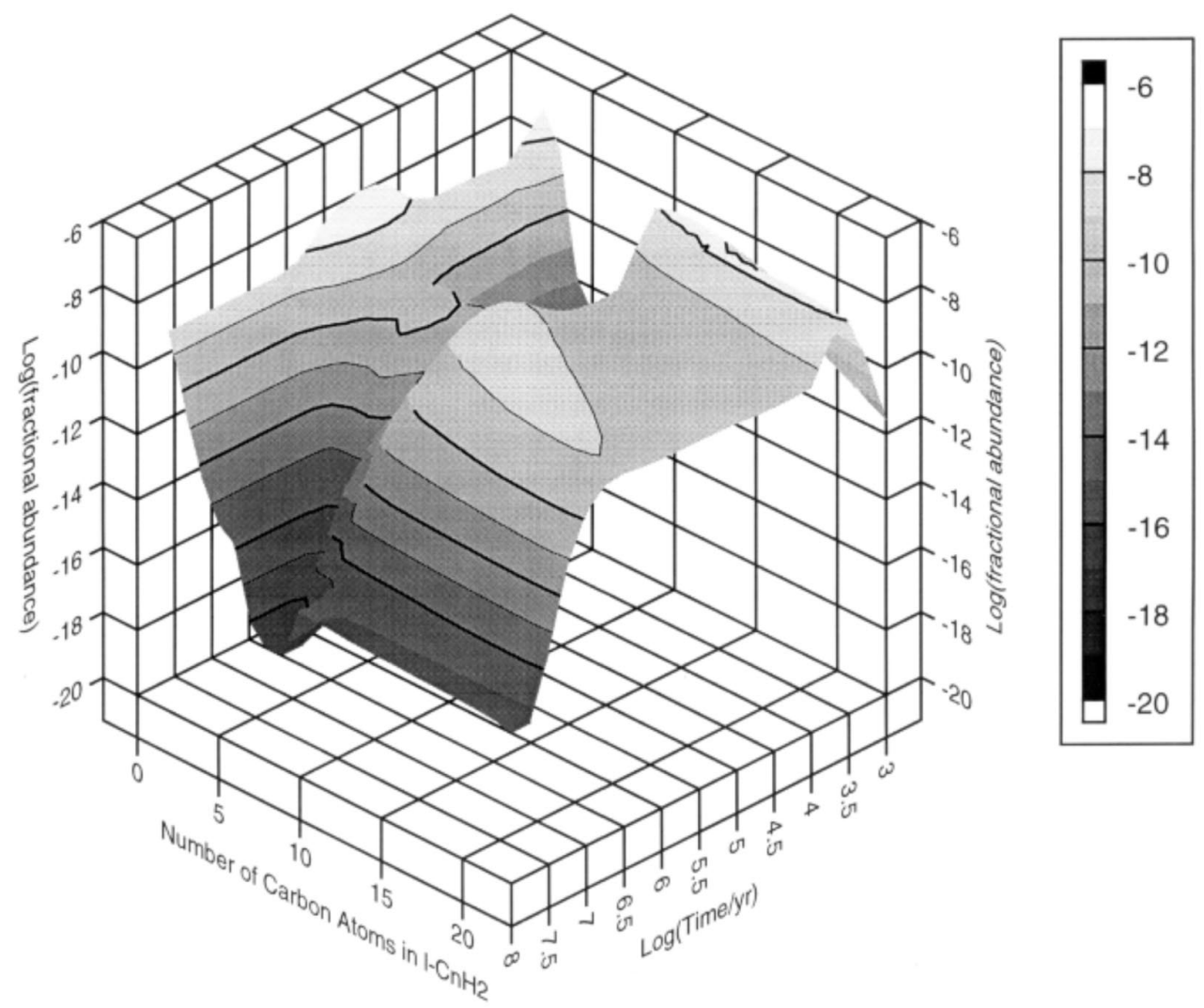

Fig. 8.-Three-dimensional plot of the fractional abundance of the linear species $1-\mathrm{C}_{n} \mathrm{H}_{2}$ vs. $n$ and time obtained with the M4 network. The gray scale is used in conjunction with the ordinate to indicate fractional abundance.

most other organic species. Moreover, the times at which maximum abundances are attained do not significantly increase with increasing molecular size.

Interestingly, the method of synthesis of the linear carbon species differs between the two models. In the NSM network, synthesis of the smaller species occurs mainly by ion-molecule insertion reactions involving carbon ions and neutral hydrocarbons, or carbon atoms and positively charged hydrocarbon ions:

$$
\begin{aligned}
\mathrm{C}^{+}+\mathrm{C}_{n} \mathrm{H}_{m} & \rightarrow \mathrm{C}_{n+1} \mathrm{H}_{m-1}^{+}+\mathrm{H}, \\
\mathrm{C}+\mathrm{C}_{n} \mathrm{H}_{m}^{+} & \rightarrow \mathrm{C}_{n+1} \mathrm{H}_{m-1}^{+}+\mathrm{H} ;
\end{aligned}
$$

whereas in the M4 network, neutral insertion reactions between $\mathrm{C}$ and hydrocarbons, e.g.,

$$
\mathrm{C}+\mathrm{C}_{n} \mathrm{H}_{m} \rightarrow \mathrm{C}_{n+1} \mathrm{H}_{m-1}+\mathrm{H},
$$

play a significant role. In both models, radiative association reactions become increasingly significant with increasing reactant size.

\section{RESULTS: RINGS AND FULLERENES}

When linear species spontaneously convert into monocyclic rings as the number of carbon atoms reaches 24 , the abundances tend to increase, since ring species are assumed to be less reactive than their linear counterparts. This effect can be seen in the three-dimensional Figures 9 and 10, in which the fractional abundances of both linear $(n<24)$ and monocyclic $(24 \leq n \leq 63)$ bare carbon clusters are plotted versus time and the number of carbon atoms $n$ for the NSM and M4 networks, respectively. In the NSM network, the jump between $n=23$ and $n=24$ is a rather small one, but in the M4 network, the jump is quite large, such that the abundance of the monocyclic $\mathrm{C}_{24}$ cluster is approximately equal to that of the linear cluster $\mathrm{C}_{9}$ at early times. As $n$ increases further, the abundances of the bare monocyclic species decrease monotonically up to $n=63$ (the special case of $n=64$ is discussed below). The time dependence of the monocyclic species through $n=63$ is not different from that of the linear species; abundances tend to decrease from early-time values as steady state conditions are reached. In the M4 results, it can be seen that the peak abundances of the largest monocyclic bare carbon species are achieved at significantly later times than the peak abundances of the linear and smaller monocyclic species.

The abundances of all tricyclic species are very low because they are produced slowly by association and condensation reactions of ionic with neutral monocyclic species, and because they are destroyed rapidly by a variety of ion-molecule reactions to form fullerenes (Bettens \& Herbst 1995; Bettens \& Herbst 1996).

As time progresses, two groups of "sink" species can become especially abundant, as also occurs in dispersive clouds. Sink species belong to groups that do not contain destruction reactions, except among members of the group. The time dependence of such species and/or groups does not show a peak at early time, but shows a continuous increase with time until a quasi steady state is achieved, at which time formation and depletion reactions only occur within the groups. Still, the early-time results are of importance because it is difficult to see how steady state conditions can be reached in realistic models of interstellar clouds given the long time necessary to reach them. One major group of sink species is composed of molecules containing 64 carbon atoms. These species, the largest in the model, 


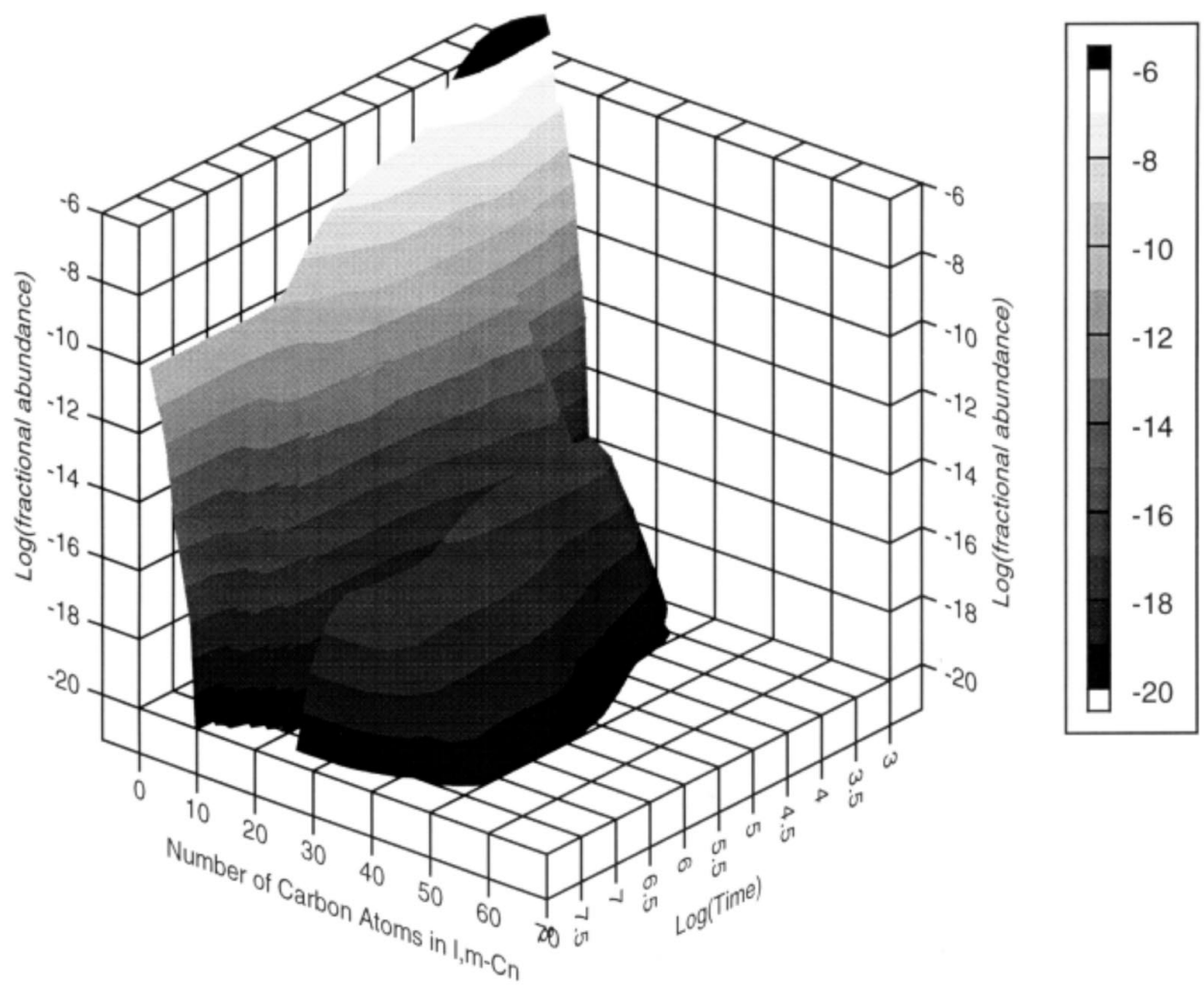

Fig. 9.-Three-dimensional plot of the fractional abundances of the linear and monocyclic bare carbon cluster species, 1- $\mathrm{C}_{n}(n<24)$ and $\mathrm{m}-\mathrm{C}_{n}$ $(24 \leq n<64)$, vs. $n$ and time obtained with the NSM network. The gray scale is used in conjunction with the ordinate to indicate fractional abundance.

consist mainly of neutral bare and weakly hydrogenated monocyclic hydrocarbons as well as the bare neutral fullerene. Since synthesis past this size is not considered, the 64 carbon atom species effectively represent species with 64 or more carbon atoms. Another group of sink species consists of the bare (and particularly stable) neutral fullerene $\mathrm{C}_{60}$ along with the much less abundant positive fullerene/ane ions $\mathrm{C}_{60} \mathrm{H}_{m}^{+}$.

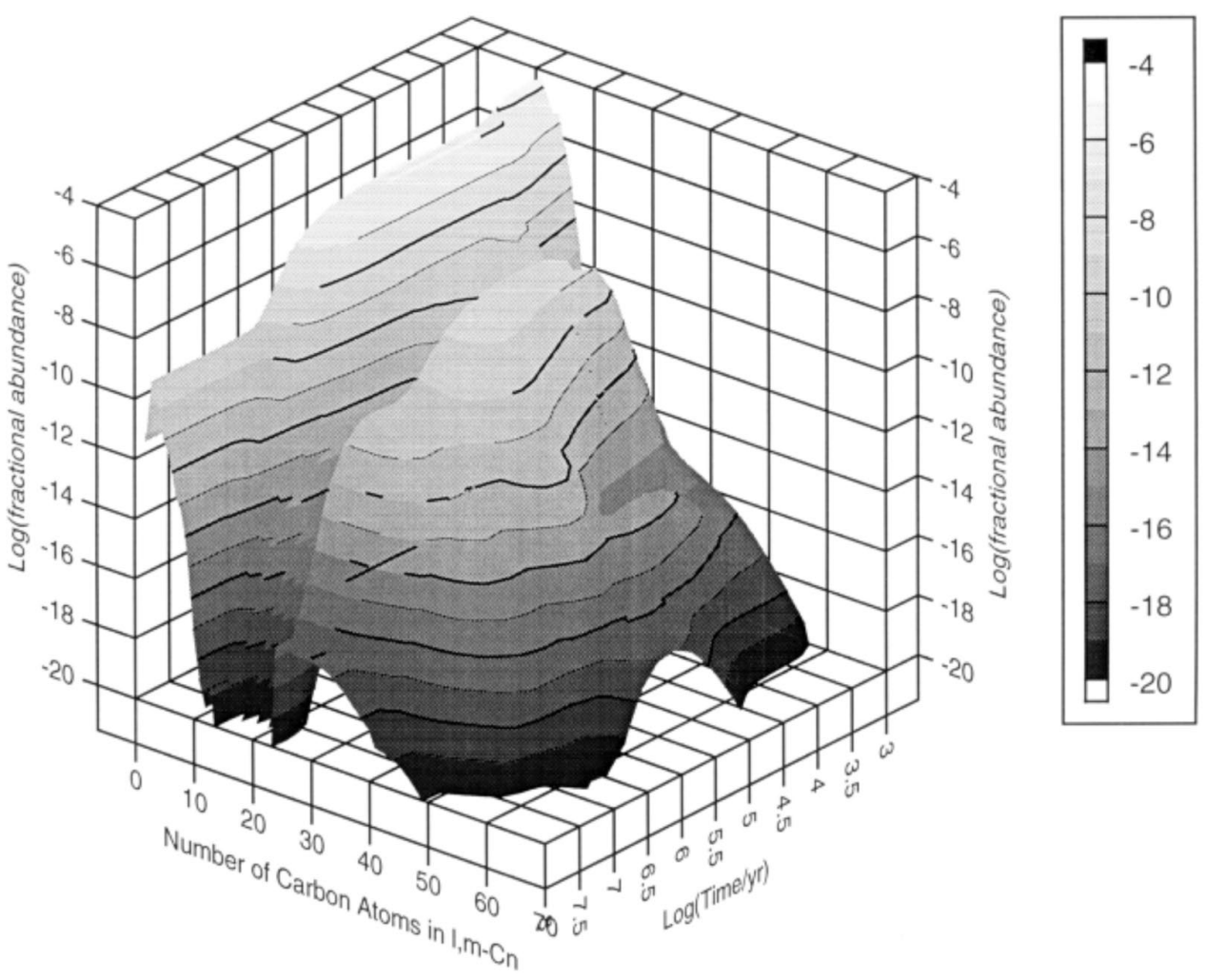

Fig. 10.-Three-dimensional plot of the fractional abundances of the linear and monocyclic bare carbon cluster species, 1- $\mathrm{C}_{n}(n<24)$ and $\mathrm{m}_{-} \mathrm{C}_{n}$ $(24 \leq n<64)$, vs. $n$ and time obtained with the M4 network. The gray scale is used in conjunction with the ordinate to indicate fractional abundance. 


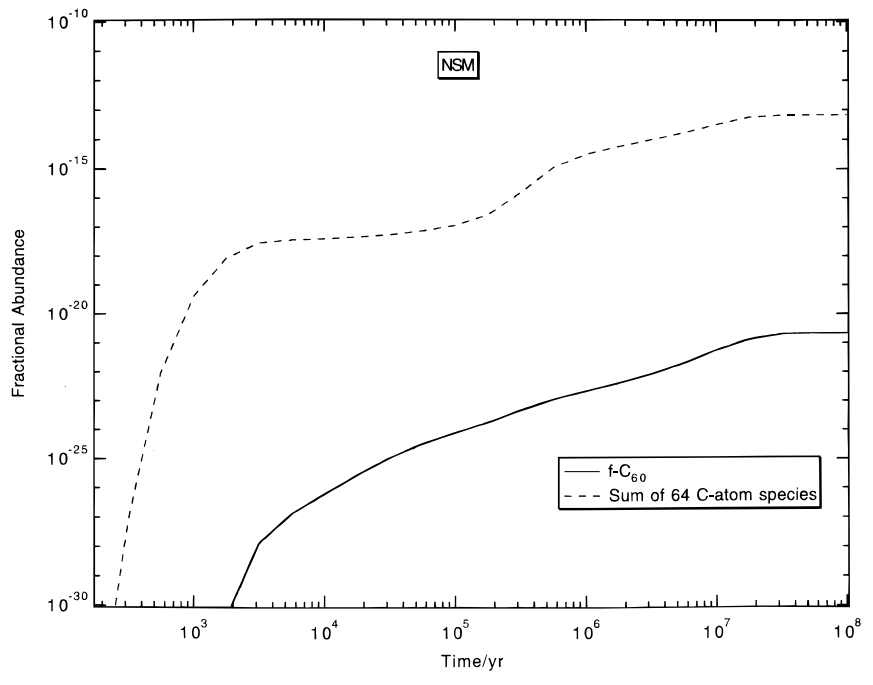

FIG. 11.-Plot of the fractional abundances of the group of 64 carbon atom species and of the fullerene $\mathrm{C}_{60}$ vs. time for the NSM network.

Figures 11 and 12 show the total fractional abundance of 64 carbon atom species and the fractional abundance of the fullerene $\mathrm{C}_{60}$ versus time for the NSM and M4 calculations, respectively. In the NSM calculations, the abundances of the two groups of sink species are very low, whereas in the M4 calculations they are found to be considerable. In particular, even at a time of $10^{5} \mathrm{yr}$, both the fullerene $\mathrm{C}_{60}$ and the group of 64 carbon atom species have calculated fractional abundances of nearly $10^{-8}$. The abundance of fullerene stands for the group abundance of both bare $\mathrm{C}_{60}$ and partially hydrogenated fulleranes $\left(\mathrm{C}_{60} \mathrm{H}_{m}\right)$, since the latter are not considered in the model because of space limitations. It is indeed likely that some hydrogenation occurs, so that the large calculated abundance must be divided among several species. Likewise, the large abundance of the group of 64 carbon atom species does not necessarily imply an observable abundance for any one species. In fact, the M4 model is so powerful synthetically that the calculated abundances may represent small dust particles with far more than 64 carbon atoms.

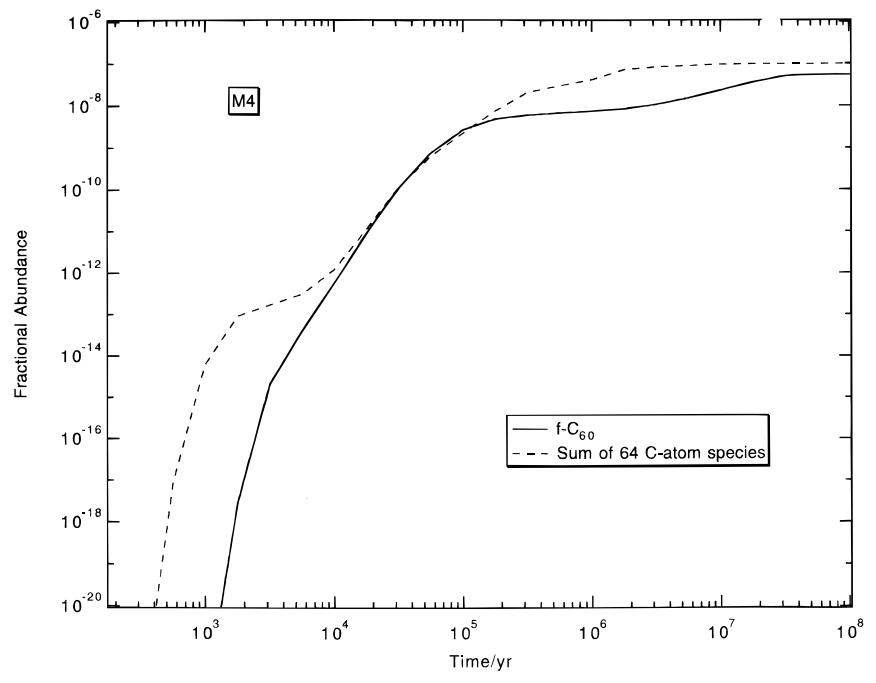

FIG. 12.-Plot of the fractional abundances of the group of 64 carbon atom species and of the fullerene $\mathrm{C}_{60}$ vs. time for the M4 network.
It is interesting to compare our dense cloud results for 64 carbon atom species and for the fullerene $\mathrm{C}_{60}$ with our corresponding dispersive cloud results (Bettens \& Herbst 1996). With the M4 network, there is very little difference in either the calculated fractional abundances or their time dependence. With the NSM network, however, the formation of these large molecules is enhanced dramatically under dispersive cloud conditions.

\section{DISCUSSION}

Calculated abundances in dense interstellar clouds for all molecules in the extended NSM and M4 networks are available from the authors. The calculated abundances for clusters and hydrocarbons with more than 10 carbon atoms, however, are highly dependent on which model network is utilized; Table 1 and the figures show that detection of these large molecules is far more feasible if the M4 network pertains. Is there any manner in which we can currently decide which network (if either) is more likely to be correct? The two networks can be compared in two ways: (1) with observations for smaller hydrocarbons and other types of smaller molecules, and (2) with the current state of knowledge of reaction rates involving neutral species.

For the well-studied cloud TMC-1, the NSM network does a better job at early time than does the M4 network (Bettens et al. 1995; Lee et al. 1996a). In a comparison involving $\mathrm{C}_{n} \mathrm{H}$ radicals, the latter network underpredicts the abundances of the $\mathrm{C}_{2} \mathrm{H}$ and $\mathrm{C}_{4} \mathrm{H}$ radicals even at their peak, but shows a smaller diminution in peak abundance with increasing molecular size. In the well-studied cyanopolyyne family $\left(\mathrm{HC}_{2 n+1} \mathrm{~N}\right)$, the M4 and NSM results show a similar contrasting pattern, although here the NSM network leads to too large a value for the smallest member of the family $\left(\mathrm{HC}_{3} \mathrm{~N}\right)$ if the peak calculated value is used. If one wishes to have a less severe time constraint on the calculated abundances for the smaller species, the NSM results are certainly superior in that they can produce sufficient abundances over larger periods of time. Of course, the comparison with observation is complicated by the probable need to account for heterogeneous physical conditions.

Although the NSM network results in better early-time agreement with observation in TMC-1, it runs more seriously afoul of recent low-temperature experimental results on neutral-neutral reactions. The M4 network takes these into account with the assumption that a wide variety of atom-neutral and radical-neutral reactions are rapid. However, this network specifically excludes the class of reactions between $\mathrm{O}$ and $\mathrm{N}$ atoms and bare carbon clusters. Although there is some theoretical evidence that $\mathrm{O}$ atoms do not react with small linear carbon clusters containing an odd number of carbon atoms (Woon \& Herbst 1996), it is far more likely that $\mathrm{O}$ atoms will react with linear carbon clusters containing an even number of carbon atoms, since these clusters possess ground electronic states of triplet multiplicity. If $\mathrm{O}-\mathrm{C}_{2 m}$ reactions are assumed to be rapid for $m>1$, the synthetic power of the resulting network is reduced (Bettens et al. 1995). Research is continuing to determine how this synthetic power can be restored so that the abundances of the larger interstellar molecules currently detected can be accounted for. One possibility is that the small $\mathrm{C}_{2 m}$ species possess nonreactive ringed isomers for $m>1$ of significant interstellar abundance. Another possibility is that hydrogenation ("HAAR") reactions between 
carbon clusters or $\mathrm{C}_{n} \mathrm{H}$ radicals and $\mathrm{H}_{2}$ can occur at low temperatures via tunneling, leading to increased synthetic power (Bettens et al. 1995). These possibilities have not yet been explored fully by theoretical and experimental studies. As such studies are undertaken, better reaction networks involving small molecules can be constructed, and extensions of these networks to large molecules will lead to a greater degree of certainty than the current results.

The best observational constraints on the predictions of the current extended networks lie perhaps in studies of larger members of the $\mathrm{C}_{n} \mathrm{H}$ and $\mathrm{HC}_{2 n+1} \mathrm{~N}$ families, although the latter species have yet to be included for $n>4$. Radio searches for these large molecules in dense interstellar clouds may well be feasible, especially given the recent rapid progress in their laboratory spectroscopy
(McCarthy et al. 1996a, 1996b; Travers et al. 1996). Large molecules in cold sources have peak emissions at rather low frequencies that we have previously estimated (Herbst 1985). For example, the optimum emission frequency for a 19 carbon atom linear chain species in a $10 \mathrm{~K}$ source is approximately $6 \mathrm{GHz}$, corresponding to the $J=70 \rightarrow 69$ transition. The emission spectra of nonlinear species peak at larger frequencies. Detection via absorption is also a possibility.

We acknowledge the support of the National Science Foundation for our research program in interstellar chemistry. We also thank the Ohio Supercomputer Center for computer time on their Cray Y-MP/8.
Bergin, E. A., Langer, W. D., \& Goldsmith, P. F. 1995, ApJ, 441, 222

Bettens, R. P. A., \& Herbst, E. 1995, Int. J. Mass Spect. Ion Proc., 149/150, 321

. 1996, ApJ, 468, 686

Bettens, R. P. A., Lee, H.-H., \& Herbst, E. 1995, ApJ, 443, 664

Giesen, T. F., Orden, A. V., Hwang, H. J., Fellers, R. S., Provencal, R. A., \&

Saykally, R. J. 1994, Science, 265, 756

Herbig, G. H. 1993, ApJ, 407, 142

Herbst, E. 1985, Origins of Life, 16, 3

. 1991, ApJ, 366, 133

1995, Ann. Rev. Phys. Chem., 46, 27

. 1996, in Atomic, Molecular, and Optical Physics Handbook, ed. G. Drake (New York: AIP), 429

Herbst, E., Lee, H.-H., Howe, D. A., \& Millar, T. J. 1994, MNRAS, 268, 335

Herbst, E., \& Leung, C. M. 1989, ApJS, 69, 271 1990, A\&A, 233, 177

Hunter, J. M., Fye, J. L., Roskamp, E. J., \& Jarrold, M. F. 1994, J. Phys. Chem., 98, 1810

\section{REFERENCES}

Le Bourlot, J., Pineau des Forêts, G., \& Roueff, E. 1995, A\&A, 297, 251

Lee, H.-H., Bettens, R. P. A., \& Herbst, E. 1996a, A\&AS, 119, 111

Lee, H.-H., Herbst, E., Pineau des Forêts, G., Roueff, E., \& Le Bourlot, J. 1996b, A\&A, 311, 690

McCarthy, M. C., Travers, M. J., Kalmus, P., Gottlieb, C. A., \& Thaddeus, P. 1996a, ApJ, 467, L125

McCarthy, M. C., Travers, M. J., Thaddeus, P., \& Gottlieb, C. A. 1996b, Ohio State University Int. Symp. on Molecular Spectroscopy, 51, 171

Sims, I. R., \& Smith, I. W. M. 1995, Ann. Rev. Phys. Chem., 46, 109

Thaddeus, P. 1994, in AIP Conf. Proc. 312, Molecules and Grains in Space, ed. I. Nenner \& L. Trojanowski (New York: AIP), 711

Travers, M. J., McCarthy, M. C., Kalmus, P., Gottlieb, C. A., \& Thaddeus, P. 1996, ApJ, 469, L65

von Helden, G., Gotts, N. G., \& Bowers, M. T. 1993, Nature, 363, 60

Woon, D. E., \& Herbst, E. 1996, ApJ, 465, 795

Xie, T., Allen, M., \& Langer, W. D. 1995, ApJ, 440, 674 